\title{
Preprocessing and Skull Stripping of Brain Tumor Extraction from Magnetic Resonance Imaging Images Using Image Processing
}

\author{
Shweta Suryawanshi ${ }^{\mathrm{a}, 1}$ and Dr. Sanjay B. Patil ${ }^{\mathrm{b}}$ \\ ${ }^{a}$ E \& TCDepartment, BSCOER, Narhe, Pune, India \\ ${ }^{b}$ Principal, Rajgad Dnyanpeeth's Shree Chhatrapati Shivajiraje College \\ of Engineering Pune, India
}

\begin{abstract}
Many neuroimaging processing functions believe the preprocessing and skull strip (SS) to be an important step in brain tumor diagnosis. For complex physical reasons intensity changes in brain structure and magnetic resonance imaging of the brain, a proper preprocessing and SS is an important part. The method of removing the skull is relayed to the taking away of the skull area in the brain for medical investigation. It is more correct and necessary techniques for distinguishing between brain regions and cranial regions and this is believed a demanding task. This paper gives detailed review on the preprocessing and traditional transition to machine learning and deep learning-based automatic SS techniques of magnetic resonance imaging.
\end{abstract}

Keywords: Diagnosis, brain tumor, magnetic resonance image, machine learning, deep learning.

\section{Introduction}

In some parts of the human body, there is an unlimited growth of tumor cancer cells. There are different types of tumors that have specific specificities and different treatments. Brain Tumors (BTs) are classified as primary BT as well as metastatic BTs. The BT tends to stay in the brain even in the early stages, and then cancer tends to move to other parts of the body as well as spread to the brain. Actually, the majority utilized rating method has been declared by the WHO. It categorizes BTs into grade I to IV using the microscope. Premature detection of BT can be life-threatening, so diagnostic procedures have changed as needed. Medically, treatment for BT consists of surgical treatment, radiation treatment, or chemotherapy [1].

With the advancement of medical imaging, the efficiency of images plays an important role in the assessment of patients with BTs, besides; there is a significant power of patient anxiety. In modern times, increasingly new imaging methods such as Magnetic Resonance Imaging show the whole characteristics of BT and clinical doctors are developing to examine the BT method as desired. Treatment for BT involves surgery, radiation treatment, or chemotherapy. Medical practitioners play an important role in BT assessment, including treatment.

\footnotetext{
${ }^{1}$ Shweta Suryawanshi, E \& TC Department, BSCOER, Narhe, Pune, India Email: suryawanshi.shweta02@gmail.com.
} 
Once BT is clinically believed, radiological assessments are made to determine the relationship between BT status, BT area, and the structure associated with it. This knowledge is essential as well as important for making decisions in a variety of treatments such as surgery, radiation treatment or chemotherapy. As a result, evaluating BT with imaging methods is one of the important aspects of radiology departments at this time [2].

Magnetic Resonance Imaging is not involving the introduction of instruments into the body with superior soft tissue contrast imaging modality, which gives very useful knowledge regarding nature, dimension, and localization of BTs not including exposing the patient to a high ionization radiation. Magnetic Resonance Imaging is attracting extra with additional interests for the BT analysis in the medical field. An axial section of four standard sequences for glioblastoma is shown in Figure 1 [3]. In current medical practice, various magnetic resonance images with explanations of tumor segments are used for success analysis. These succession images include T1Weight Magnetic Resonance Imaging (T1w), T1-Weighted Magnetic Resonance Imaging through Contrast Enhancement (T1wc), T2-Weighted Magnetic Resonance Imaging, Proton Density-weighted Magnetic Resonance Imaging, Fluid- Attenuated Inversion Recovery (FLAIR), etc.

Currently, a large number of BT images are being created in health centers, as physicians can't interpret these images promptly [4]. Therefore, automatic segmentation estimates have been developed. BT segmentation is the edema of regular brain tissue with regular cells, necrotic core and white matter (WM), gray matter (GM), and cerebrospinal fluid (CSF) [5]. Segmentation of irregular tissues in the segment including machine learning, medical imaging has created significant developments in the field of BT segmentation [6]. In general, most irregular BT tissues can be directly identified by the BT segmentation technique. However, precisely not all systems with reproductive partitioning effects, including instances of deformation, are resolved. BT segmentation has a huge impact on identification, monitoring, action schedules for patients as well as medical tests.

This paper shows detailed review on the preprocessing and traditional transition to machine learning and deep learning-based automatic SS techniques of magnetic resonance imaging.

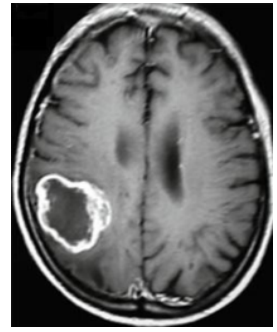

(a) T1-weighted MRI

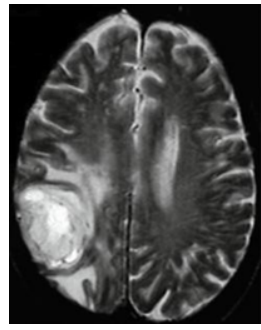

(b) T2-weighted MRI 


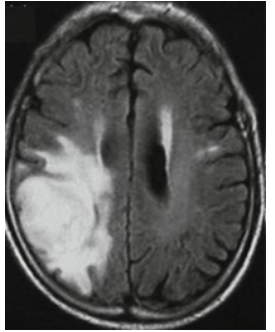

(c) FLAIR

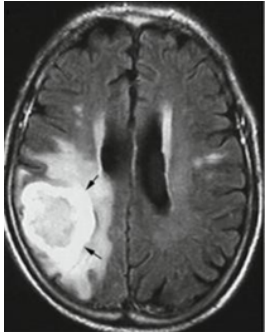

(d) FLAIR with contrast

Figure 1. Four imaging modalities [3]

\section{Preprocessing}

Before the Brain Tumor Segmentation (BTS) methods, the Magnetic Resonance Imaging preprocessing actions are initiated as it is straightforwardly relayed to the superiority of the segmentation outcomes [7]. Generally, the untreated Magnetic Resonance Imaging images require preprocessing to recognize BTS purposes. Such pre-processing functions consist of filtering, skull-stripping, etc, as well as have straight effect on the effects of BTS. Image filtering is a typical preprocessing part for Magnetic Resonance Imaging [8].

The term noise is used to denote any random attenuation of a signal and is related to images. Noise is sent by image so noise can come through image acquirement or could result from a noisy communication channel over which the image is sent [7]. Either way, the denoising job is to utilize the knowledge that have on the statistical structure of images to eliminate the result of the noise as well as probable. One of the main difficulties facing in the Image Processing (IP) is image denoising. There are several filtering techniques existing. The wide classification of different image denoising techniques is provided in the Figure 2.

In [9], provides a behavioral analysis of the Anisotropic Diffusion Model (ADM) of the authors Perona and Malik. The main proposition was to tell the equation from the difficulty of some optimization to find the reality of the unique global minimum reality to maintain the existence of some dense global minima. Also, the smooth, as well as the edge enhancing method of anisotropic diffusion, is shown during the eigenvalue disintegration of the diffusion equation.

Two wavelet-domain filtering (WDF) techniques were given to reduce noise in magnetic resonance imaging [10]. The wavelet coefficient with the corresponding thresholding technique [11] is not different from the previously suggested technique for magnetic resonance imaging evaluation based on soft thresholding [12]. To handle this extra difficult situation, a WDF was obtained that works on an image of square magnitude.

The new Noise-Adaptive Method (NAM) verified the reversal of certain filters when incredible unreliable noise was occurring in magnetic resonance imaging [13]. Most filtering magnetic resonance imaging relies on the same distribution over noise distribution. Since this prediction is not true, the resulting filtering suit is suboptimal. This is the case with magnetic resonance imaging with local unrealistic noise levels, for 
example, obtained by parallel imaging. An imaginary technique where knowledge of the noise level of a local image is used to reduce the power of the filter. This knowledge is obtained involuntarily from images through new local sound assessment technology. This technique was standardized as well as evaluated with sample nonlocal i.e. filters with authentic magnetic resonance imaging data that saw enhanced rewards in all conditions.

The Independent Component Analysis (ICA) has proven immense promise for extracting feature information, basically deciding the building blocks of each provided data [14]. ICA is a statistical method that seeks to find examples of empirical data as the components are as independent as possible. The ICA is applied to the image data to identify the morphology in the image and the ICA gives a representation. That representation can be considered the primary structure in this data. ICA is a highly novel data-analysis tool that has a huge promise to judge the relevant specific features in high-dimensional data.

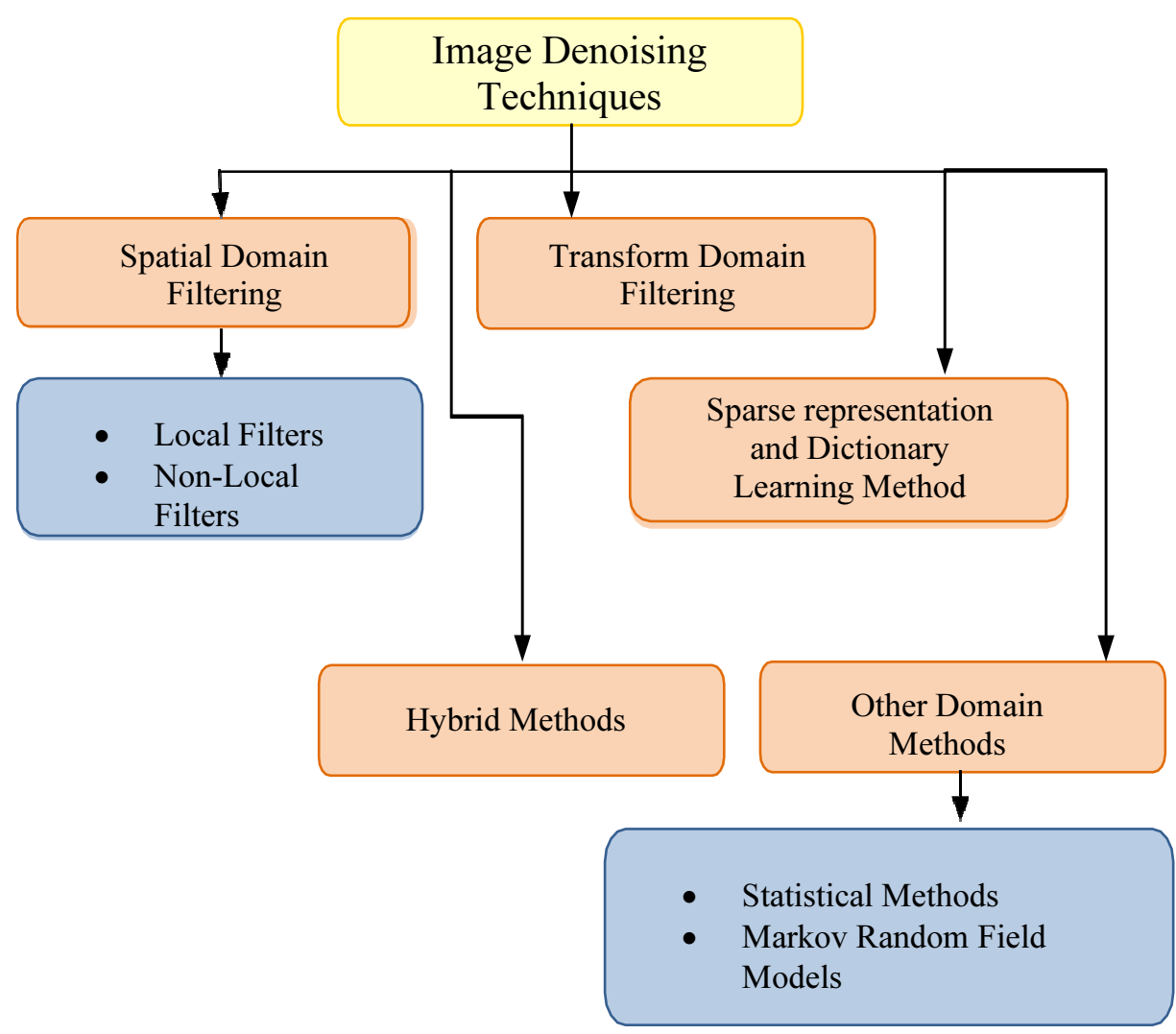

Figure 2. Image Denoising Techniques

It is written in the literature that noise can be reduced in magnetic resonance images by using numerous techniques. The purpose of the paper [15] is to critique modern de- 
noising algorithms based on statistical assumptions and their ability to improve the outcome of brain tumor segmentation. ADM is currently the most widely accepted technique for De-noised of BTs magnetic resonance imaging. Although the noise of the images has decreased, it has been suggested that it has persisted permanently as well as turned into a negative result of BT segmentation.

\section{Skull Stripping}

$\mathrm{SS}$ is believed one of the dangerous preprocessing methods that should help in the accurate identification of BT. SS also reduces the likelihood of incorrect classification of brain tissues for periods of the division with incompatible tissues. Accurate brain extraction improves the likelihood of self-diagnosis of numerous neurological disorders such as dementia, as well as schizophrenia. The main idea of SS is that it removes nonbrain tissue-dura substance just like the external blood vessels, as well as is lost only in the area of the brain. These non-brain tissues enhance the computational effectiveness of various neuroimaging algorithms. $S S$ is categorized into various groups as given below.

- Manual,

- Semi-Automated,

- Automated Methods.

The advances increased for SS or brain Magnetic Resonance Imaging extraction has been classified into three main Classes.

1) Conventional SS (CSS): Mathematical operations.

2) Machine-learning-based SS (MLSS): Fuzzy Logic (FL), Support Vector Machines (SVMs), Artificial Neural Networks (ANNs), Bayesian Classifiers (BCs)

\section{3) Deep-learning-based SS (DLSS): use of Convolution Neural Network}

(CNN), Input Parameters (IPs) and Hidden Layers (HLs).

CSS advances use traditional or regular methods to reach SS. This section covers the content of the usual IP techniques with most SS techniques. The classification of CCS techniques is shown in Table. 1.

Table 1. Conventional skull stripping techniques

\begin{tabular}{|l|l|l|l|}
\hline Types & Methodology & Discussion & \multicolumn{1}{|c|}{ Results } \\
\hline $\begin{array}{l}\text { Deformable } \\
\text { Surface-Based } \\
\text { Skull Stripping } \\
\text { Methods (DSSS) } \\
\text { [16]. }\end{array}$ & $\begin{array}{l}\text { The (DSSS) method primarily } \\
\text { says the surface model with } \\
\text { frequently distorts the surface in } \\
\text { anticipation of it create the best } \\
\text { possible result. }\end{array}$ & $\begin{array}{l}\text { This technique does } \\
\text { not involve any pre- } \\
\text { processing method. }\end{array}$ & $\begin{array}{l}\text { It is extremely robust as well } \\
\text { as precise. }\end{array}$ \\
\hline
\end{tabular}




\begin{tabular}{|c|c|c|c|}
\hline $\begin{array}{l}\text { Mathematical } \\
\text { Morphological } \\
\text { Operation-based } \\
\text { Skull Stripping } \\
\text { Methods } \\
\text { (MMOSS)[17]. }\end{array}$ & $\begin{array}{l}\text { Effort through Thresholding with } \\
\text { morphological erosion and } \\
\text { dilation actions in sequence. }\end{array}$ & $\begin{array}{l}\text { One of the drawbacks } \\
\text { is that they are } \\
\text { parameter needy for } \\
\text { example edge constant, } \\
\text { diffusion iteration, } \\
\text { diffusion, as well as } \\
\text { erosion size. }\end{array}$ & $\begin{array}{l}\text { Average performance of } \\
\text { method } 94.3 \% \text {. }\end{array}$ \\
\hline $\begin{array}{l}\text { Intensity-Based } \\
\text { Skull Stripping } \\
\text { (IbSS) Methods } \\
{[18] .}\end{array}$ & $\begin{array}{l}\text { IbSS advances on modeling } \\
\text { intensity sharing of brain } \\
\text { Magnetic Resonance Imaging } \\
\text { utilized for threshold } \\
\text { categorization. }\end{array}$ & $\begin{array}{l}\text { IBSS techniques are } \\
\text { responsible for } \\
\text { accelerating their } \\
\text { smoothness; However, } \\
\text { their accuracy can vary } \\
\text { in different datasets } \\
\text { with incredible levels of } \\
\text { image resolution, } \\
\text { sound and artwork. }\end{array}$ & $\begin{array}{l}\text { Significantly progress the } \\
\text { clinical efficiency. }\end{array}$ \\
\hline $\begin{array}{l}\text { Template-Based } \\
\text { Skull Stripping } \\
\text { (TSS) Methods. } \\
\text { [19]. }\end{array}$ & $\begin{array}{l}\text { TSS or Atlas-based approaches } \\
\text { of SS rely on pattern or atlas on } \\
\text { Magnetic Resonance Imaging of } \\
\text { brain to divide them from non- } \\
\text { brain tissues. Making an early } \\
\text { estimate for the brain mask } \\
\text { (BM), the BM border is } \\
\text { segmented once more by a } \\
\text { classifier, which improves the } \\
\text { last result precision. }\end{array}$ & $\begin{array}{l}\text { Fiducially marker } \\
\text { registration based on } \\
\text { the maxillary template } \\
\text { is accurate enough for } \\
\text { image-directed surgery } \\
\text { at the base of the } \\
\text { ancestral skull, but not } \\
\text { for the lateral skull } \\
\text { base. }\end{array}$ & $\begin{array}{l}\text { TSS is a precise however } \\
\text { noninvasive registration } \\
\text { technique for anterior skull } \\
\text { base surgical treatment. }\end{array}$ \\
\hline $\begin{array}{l}\text { Hybrid-Based } \\
\text { Skull Stripping } \\
\text { (HSS) Methods } \\
{[20] .}\end{array}$ & $\begin{array}{l}\text { The mixture of further than one } \\
\text { technique from earlier existing } \\
\text { SS techniques. }\end{array}$ & $\begin{array}{l}\text { HSS merges various } \\
\text { techniques of SS } \\
\text { algorithms that } \\
\text { compose them to work } \\
\text { jointly to attain an } \\
\text { improved solution to a } \\
\text { difficulty. }\end{array}$ & Shows high accuracy. \\
\hline
\end{tabular}

To get practical results, CSS methods need to be modified in different numerical parameters based on the dataset, where MLSS methods are increasing to get more successful results. The classification of MLSS techniques is shown in Table. 2. Deep learning (DL) with IP and HL is done by CNN. In an in-depth study of DL, as with normal $\mathrm{NN}$, each input of magnetic resonance imaging exceeds the sequence of convolution layers. The different skull stripping algorithms based on CNN are proposed in [21] - [30]. Therefore, it is a challenging task to create SS every time the brain images have different contrasts, scans quality, and intensity. Different SS algorithms are proposed namely manual, semi- automatic, and automated algorithms. Automatic SS greatly improves the accuracy with effectiveness of neuroimaging 
algorithms. The results of novel DL based skull stripping algorithms are more accurate and precise than usual presented methods.

Table 2. Machine Learning Based skull stripping techniques

\begin{tabular}{|c|c|c|c|}
\hline Types & Methodology & Discussion & Results \\
\hline $\begin{array}{l}\text { Model-Based } \\
\text { Level Set } \\
{[31] .}\end{array}$ & $\begin{array}{l}\text { Based on the intensity } \\
\text { dissimilarity of various } \\
\text { brain divisions with } \\
\text { curvatures of the brain } \\
\text { planes. }\end{array}$ & $\begin{array}{l}\text { Entailed postoperative } \\
\text { information to assess the } \\
\text { compassion of the system to } \\
\text { abnormal structure. }\end{array}$ & $\begin{array}{l}\text { Robust SS outcomes, creation it } \\
\text { a hopeful device for utilize in } \\
\text { large, multi- institutional, } \\
\text { population-based neuroimaging } \\
\text { learning. }\end{array}$ \\
\hline $\begin{array}{l}\text { Fuzzy } \\
\text { Logic[32]. }\end{array}$ & $\begin{array}{l}\text { Approximate the intensity } \\
\text { allocations by the utilize } \\
\text { of a priori information } \\
\text { depending leading the } \\
\text { Bayesian organization and } \\
\text { Gaussian mixture } \\
\text { form. The previous } \\
\text { information is explained } \\
\text { by fuzzy membership } \\
\text { functions. }\end{array}$ & Require prior knowledge. & $\begin{array}{l}\text { The mean sensitivity is } 98.84 \\
\% \text {, with false- positive rate was } \\
1.21 \% \text {. }\end{array}$ \\
\hline $\begin{array}{l}\text { Support } \\
\text { Vector } \\
\text { Machine with } \\
\text { local and } \\
\text { global } \\
\text { features [33]. }\end{array}$ & $\begin{array}{l}\text { An arrangement of local } \\
\text { with global knowledge is } \\
\text { utilized. The concluding } \\
\text { is required to differentiate } \\
\text { between bones moreover } \\
\text { air. }\end{array}$ & $\begin{array}{l}\text { Arrange of a little minutes } \\
\text { which is capable. }\end{array}$ & $\begin{array}{l}\text { In an association with two } \\
\text { additional techniques, one based } \\
\text { on MMOSS and the other on } \\
\text { DRSS, the SVMSS was found } \\
\text { constantly improved } \\
\text { segmentations. }\end{array}$ \\
\hline \begin{tabular}{|l} 
Principal \\
Component \\
Analysis- \\
based model \\
{$[34]$.}
\end{tabular} & $\begin{array}{l}\text { Common tissue form is } \\
\text { incarcerated by PCA } \\
\text { pathologies are } \\
\text { incarcerated by an entire } \\
\text { variation term, the skull } \\
\text { with neighboring tissue is } \\
\text { incarcerated by a lightly } \\
\text { term. }\end{array}$ & $\begin{array}{l}\text { Require of formulations for } \\
\text { various image series otherwise } \\
\text { modalities. }\end{array}$ & $\begin{array}{l}\text { Best median (97.11) and mean } \\
\text { (96.88) Dice scores over all } \\
\text { datasets. }\end{array}$ \\
\hline FLAIR [35] . & \begin{tabular}{|l|} 
Effectively able to \\
achieve using a random \\
forest of trees classifier to \\
decide with high precision \\
with rapidity whether a \\
pixel is element of the \\
brain tissue or else not.
\end{tabular} & $\begin{array}{l}\text { Superior ventricle and skull } \\
\text { size unpredictability. }\end{array}$ & $\begin{array}{l}\text { An extremely superior mean } \\
\text { dice score of } 97.02 \% \text {. }\end{array}$ \\
\hline
\end{tabular}




\section{Conclusion}

Currently, a large number of BT images are being created in health centers, as physicians can't interpret these images promptly. Therefore, automatic brain tumor diagnosis has been developed. This paper gives detailed review on the preprocessing and traditional transition to machine learning and deep learning- based automatic skull Stripping techniques of magnetic resonance imaging. The preprocessing process with the up-to-date methods of magnetic resonance imaging is reviewed. The Magnetic Resonance Imaging preprocessing actions are initiated as it is straightforwardly relayed to the superiority of the segmentation outcomes. Different SS algorithms are proposed namely manual, semi-automatic, and automated algorithms. Automatic SS greatly improves the accuracy with effectiveness of neuroimaging algorithms. The results of novel DL based skull stripping algorithms are more accurate and precise than usual presented methods.

\section{References}

[1] H. Chen, Z. Qin, Y. Ding, L. Tian, and Z. Qin, "Brain tumor segmentation with deep convolutional symmetric neural network," Neurocomputing, vol. 392, no. xxxx, pp. 305-313, 2020.

[2] J. Y. Zhang et al., "Quantitative Improvement in Brain Tumor MRI Through Structured Reporting (BTRADS)," Acad. Radiol., pp. 1-5, 2019.

A. Drevelegas, "Imaging of brain tumors with histological correlations," Imaging Brain Tumors with Histol. Correl., pp. 1-432, 2011.

[3] N. F. Interviews et al., "Significant Moments the Chair," no. 25, 2020.

[4] M. J. Pallod, "Survey on Brain Tumor Detection," pp. 1-6, 2020.

[5] G. Du, X. Cao, J. Liang, X. Chen, and Y. Zhan, "Medical image segmentation based on U-Net: A review," J. Imaging Sci. Technol., vol. 64, no. 2, pp. 1-12.

[6] Z. Ahmed, K. Mohamed, S. Zeeshan, and X. Q. Dong, "Artificial intelligence with multi-functional machine learning platform development for better healthcare and precision medicine," Database (Oxford)., vol. 2020, pp. 1-35, 2020.

[7] J. Li et al., "Automatic fetal brain extraction from 2D in utero fetal MRI slices using deep neural network," Neurocomputing, vol. 378, no. xxxx, pp. 335-349, 2020.

[8] Y. L. You, W. Xu, A. Tannenbaum, and M. Kaveh, "Behavioral analysis of anisotropic diffusion in image processing," IEEE Trans. Image Process., vol. 5, no. 11, pp. 1539-1553, 1996.

[9] R. D. Nowak, "Wavelet-based Rician noise removal for magnetic resonance imaging," IEEE Trans. Image Process., vol. 8, no. 10, pp. 1408-1419, 1999.

[10] M. Hilton, T. Ogden, D. Hattery, G. Eden, and B. Jawerth, "Wavelet Denoising of Functional MRI Data," WAVELETS Med. Biol., no. December 2014, pp. 93-114, 2019.

[11] C. S. Anand and J. S. Sahambi, "MRI denoising using bilateral filter in redundant wavelet domain," IEEE Reg. 10 Annu. Int. Conf. Proceedings/TENCON, 2008, doi: 10.1109/TENCON.2008.4766742.

[12] J. V. Manjón, P. Coupé, L. Martí-Bonmatí, D. L. Collins, and M. Robles, "Adaptive non-local means denoising of MR images with spatially varying noise levels," J. Magn. Reson. Imaging, vol. 31, no. 1, pp. 192-203, 2010.

[13] P. Hoyer, "Independent component analysis in image denoising," Master's Thesis, Helsinki Univ. Technol., no. April 1999, 1999.

[14] Diaz, P. Boulanger, R. Greiner, and A. Murtha, "A critical review of the effects of de-noising algorithms on MRI brain tumor segmentation," Proc. Annu. Int. Conf. IEEE Eng. Med. Biol. Soc. EMBS, pp. 3934 3937, 2011.

[15] S. M. Smith, "Fast robust automated brain extraction," Hum. Brain Mapp., vol. 17, no. 3, pp. 143-155, 2002 .

A. E. K. Isselmou, S. Zhang, and G. Xu, "A Novel Approach for Brain Tumor Detection Using MRI Images,” J. Biomed. Sci. Eng., vol. 09, no. 10, pp. 44-52, 2016.

[16]Z. Yang et al., "A web-based brain metastases segmentation and labeling platform for stereotactic radiosurgery," Med. Phys., pp. 1-14, 2020, doi: 10.1002/mp.14201.

[17] G. Eggers and J. Mühling, "Template-based registration for image-guided skull base surgery," Otolaryngol. - Head Neck Surg., vol. 136, no. 6, pp. 907-913, 2007, doi: 10.1016/j.otohns.2006.12.021. 
[18] S. S. and C. S. Pawar, "An Automatic Brain Tumor Detection and Segmentation using Hybrid Method," Int. J. Appl. Inf. Syst., vol. 11, no. 9, pp. 6-11, 2017, doi: 10.5120/ijais2017451641.

[19]Zhuang, J. Yang, L. Gu, and N. Dvornek, "Shelfnet for fast semantic segmentation," Proc. - 2019 Int. Conf. Comput. Vis. Work. ICCVW 2019, pp. 847-856, 2019, doi: 10.1109/ICCVW.2019.00113.

[20] H. Chen, Q. Dou, L. Yu, J. Qin, and P. A. Heng, "VoxResNet: Deep voxelwise residual networks for brain segmentation from 3D MR images," Neuroimage, vol. 170, no. April 2017, pp. 446-455, 2018, doi: 10.1016/j.neuroimage.2017.04.041.

[21] Kamnitsas et al., "Efficient multi-scale 3D CNN with fully connected CRF for accurate brain lesion segmentation," Med. Image Anal., vol. 36, pp. 61-78, 2017, doi: 10.1016/j.media.2016.10.004.

[22] O. Lucena, R. Souza, L. Rittner, R. Frayne, and R. Lotufo, "Silver standard masks for data augmentation applied to deep-learning-based skull- stripping," Proc. - Int. Symp. Biomed. Imaging, vol. 2018-April, no. Isbi, pp. 1114-1117, 2018, doi: 10.1109/ISBI.2018.8363766.

[23] O. Lucena, R. Souza, L. Rittner, R. Frayne, and R. Lotufo, "Convolutional neural networks for skullstripping in brain MR imaging using silver standard masks," Artif. Intell. Med., vol. 98, no. August 2018, pp. 48-58, 2019, doi: 10.1016/j.artmed.2019.06.008.

[24] O. Lucena, R. Souza, L. Rittner, R. Frayne, and R. Lotufo, "Convolutional neural networks for skullstripping in brain MR imaging using silver standard masks," Artif. Intell. Med., vol. 98, pp. 48-58, 2019, doi: 10.1016/j.artmed.2019.06.008.

[25] N. Imaging and L. Angeles, "Auto-context and Its Application to High- level Vision Tasks and 3D Brain Image Segmentation," IEEE Trans. Pattern Anal. Mach. Intell., vol. 32, no. 10, pp. 1744-1757, 2010, doi: 10.1109/CVPR.2008.4587436.

[26] N. H. M. Duy, N. M. Duy, M. T. N. Truong, P. T. Bao, and N. T. Binh, "Accurate brain extraction using Active Shape Model and Convolutional Neural Networks," 2018.

[27]B. Yilmaz, A. Durdu, and G. D. Emlik, "A new method for skull stripping in brain MRI using multistable cellular neural networks," Neural Comput. Appl., vol. 29, no. 8, pp. 79-95, 2018.

[28] P. Ganaye and I. C. Umr, Semi-supervised Learning for Segmentation Under Semantic Constraint Pierre-Antoine, vol. 1, no. August 2019. Springer International Publishing, 2018.

[29] H. Zhuang, D. J. Valentino, and A. W. Toga, "Skull-stripping magnetic resonance brain images using a model-based level set," Neuroimage, vol. 32, no. 1, pp. 79-92, 2006, doi: 10.1016/j.neuroimage.2006.03.019.

[30] S. Kobashi et al., "Fuzzy-ASM Based Automated Skull Stripping Method from Infantile Brain MR Images," pp. 632-632, 2008.

[31] J. Sjolund, A. E. Jarlideni, M. Andersson, H. Knutsson, and H. Nordstrom, "Skull Segmentation in MRI by a Support Vector Machine Combining Local and Global Features," Proc. - Int. Conf. Pattern Recognit., pp. 3274- 3279, 2014.

[32]X. Han et al., "Brain extraction from normal and pathological images: A joint PCA/ImageReconstruction approach,” Neuroimage, vol. 176, pp. 431-445, 2018. 